\title{
"Cup-like" blasts and NPM1 and FLT3 (ITD) mutations in acute myeloid leukemia (AML)
}

\author{
Preetesh Jain • Francisco Vega-Vazquez • \\ Stefan Faderl
}

Received: 9 March 2013/Revised: 1 April 2013/Accepted: 10 April 2013/Published online: 21 May 2013

(C) The Japanese Society of Hematology 2013

A 68-year-old male presented with progressive fatigue, weakness and respiratory infections since last few days. A complete blood cell count showed anemia (hemoglobin $9 \mathrm{~g} / \mathrm{dL})$, hyperleukocytosis $(139.3 \mathrm{~K} / \mu \mathrm{L})$ and low platelets $(50 \mathrm{~K} / \mu \mathrm{L})$ with $95 \%$ blasts in peripheral blood. Bone marrow aspirate demonstrated $93 \%$ blasts which were positive for myeloperoxidase and showed normal male (46, $\mathrm{XY})$ karyotype. These blasts were intermediate in size with folded nuclei (Fig. 1a; 500× magnification), many with a nuclear invagination giving a cup-like nuclear indentation (see arrows), or "fish-mouth" appearance. Auer rods were not seen. Figure $1 \mathrm{~b}$ from bone marrow aspirate smear demonstrates high resolution $(1000 \times$ magnification) of these "cup-like" blasts. Flow cytometry studies demonstrate that the blasts were positive for CD33, CD13, CD117 and MPO confirming the diagnosis of acute myeloid leukemia (AML). The unusual morphology of blasts with cuplike nuclear indentation, also known as a fish-mouth shape, has been described in a subset of AML with NPM1 and FLT3 (ITD) mutations. Altered nucleocytoplasmic shuttling and accumulation of mitochondria due to increased flexibility of nuclear envelope in NPMI mutations is proposed to be responsible for this alteration in the morphology of blasts.

P. Jain $(\bowtie) \cdot$ F. Vega-Vazquez $\cdot$ S. Faderl

MD Anderson Cancer Center, University of Texas,

Houston, TX, USA

e-mail: pjain@mdanderson.org

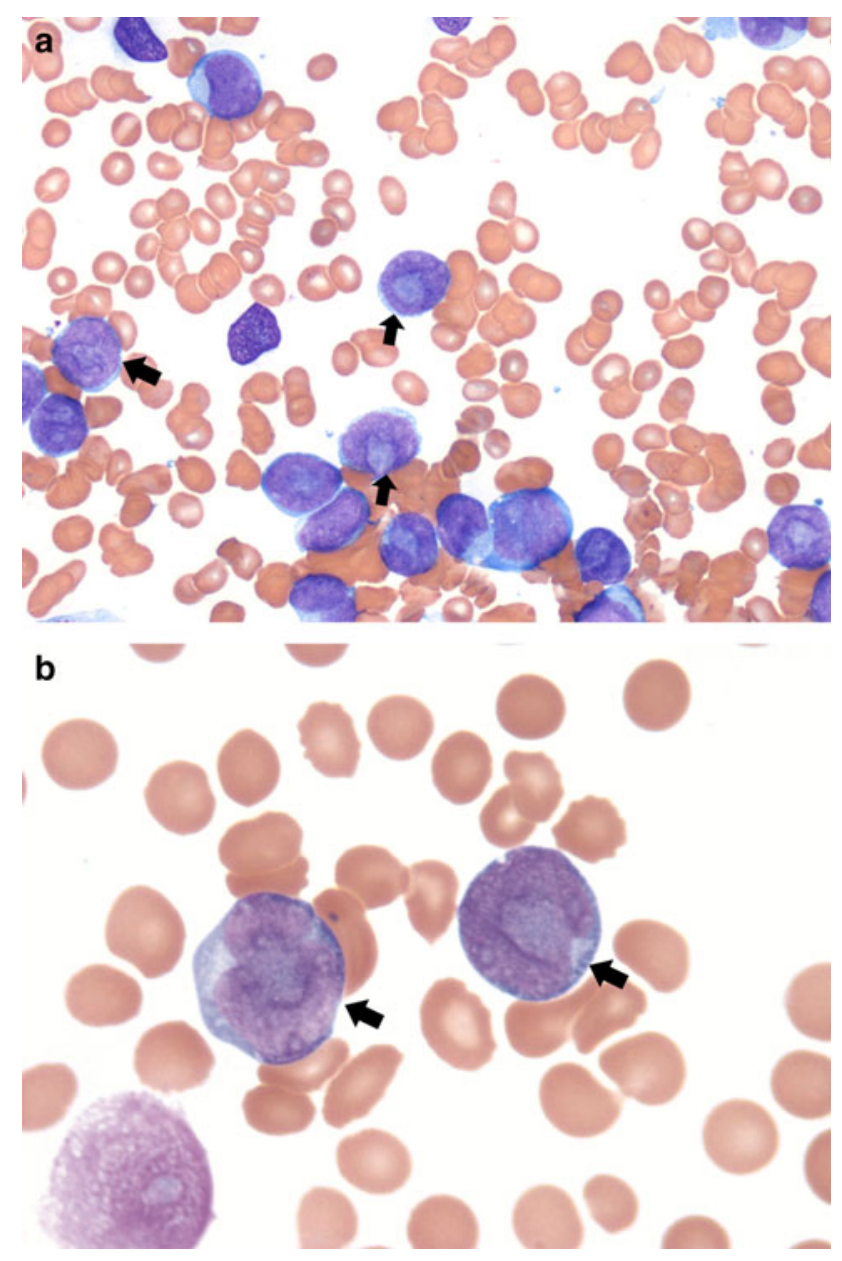

Fig. 1 a, b Bone marrow aspirate showing blasts with cup like indentation and "Fish mouth nuclei" (see arrows) 